\title{
Simulationstraining in der Geburtshilfe - lohnt sich der zeitliche Aufwand?
}

\author{
Prof. Dr. med Franz Kainer, Nürnberg
}

\section{Seit einigen Jahren gibt es die Möglichkeit, geburtshilfliche Notfallsituationen in einem Simulationskreißsaal zu trainieren. Welche Erfahrungen wurden mit diesem Trainings- modell in der Praxis gemacht? Ist ein Simulationskreißsaal sein Geld wert?}

\begin{abstract}
Notfälle im Kreißsaal führen zu einem dramatischen Anstieg der perinatalen Morbidität und Morbidität. So ist bei einer vorzeitigen Plazentalösung, einem Nabelschnurvorfall oder einer Uterusruptur eine möglichst rasche Therapie erforderlich, um katastrophale kindliche Entwicklungsstörungen $\mathrm{zu}$ vermeiden. Aber auch die Gebärende ist in vielen Fällen durch Notfallsituationen lebensbedrohlich gefährdet. So ist bei einer Fruchtwasserembolie, einer Leberruptur beim HELLP-Syndrom oder einer schweren postpartalen Blutung der Krankheitsverlauf meist ganz wesentlich vom kompetenten Ablauf der Therapiemaßnahmen abhängig.
\end{abstract}

Erschwerend kommt in diesen Situationen hinzu, dass viele Erkrankungen so selten sind, dass viele Teams den Notfall (z.B. eine Fruchtwasserembolie) das erste Mal gemeinsam erleben und daher wenig praktische klinische Erfahrung mit dem Ablauf der Therapiemaßnahmen haben. Die gute Nachricht lautet:

\section{Die meisten Fehler, die in der} Betreuung von Notfallsituationen gemacht werden, entstehen nicht aufgrund von fachlicher Unzulänglichkeit, sondern haben ihre Hauptursache in einer unzureichenden Zusammenarbeit des betreuenden Teams.

Diese sogenannten „non-technical skills“, wie Unklarheiten in der Rollen- und Aufgabenverteilung, fehlende oder mangelhafte Kommunikation und unzureichende Priorisierung bei der Ausführung von klinischen Tätigkeiten spielen in der Fehleranalyse eine entscheidende Rolle.

Das Erlernen der richtigen Kommunikation zwischen Gynäkologen, Hebammen, Pädiatern und Anästhesisten ist eine entscheidende Grundvoraussetzung, um diese Notfallsituation zu meistern.

Diese Zusammenarbeit kann trainiert werden und es gibt ausreichend Daten die belegen, dass dieses Training absolut sinnvoll ist (1-3).

Da Notfallsituationen in der Flugmedizin meist katastrophale Folgen haben, hat das Training von Notfällen in der Luftfahrt eine lange Tradition. Erschwerend für das betreuende Flugpersonal kommt ja bei einem Flugunfall hinzu, dass auch das Team mit dem Piloten beim Absturz gefährdet ist. Beim geburtshilflichen Notfall trifft die körperliche Schädigung oder der Tod primär das Kind oder die Mutter und nicht das Team. Das mag vielleicht mit ein Grund sein, warum die optimale Umsetzung von Notfällen in der Geburtshilfe im Vergleich zur Flugindustrie noch in den Kinderschuhen steckt.

Das Lernen aus Fehlern in Notfallsituationen war in der Vergangenheit auch dadurch erschwert, dass es keine effiziente Kultur der Fehleranalyse gab. Fehler wurden meist Personen zugeordnet, die in der hierarchischen Klinikstruktur an der untersten Stufe standen. Man hatte dann meist einen Schuldigen gefunden, den man bei dramatischen Fehlern dann austauschen konnte, um eine Konsequenz gezogen zu haben. Da man jedoch die Hauptursache, die fehlerhafte Kommunikation, nicht verbessert hat, war es nur eine Frage der Zeit, bis der Fehler wieder aufgetreten ist.

Die Ziele eines Simulationstrainings in der Geburtshilfe, das die Sicherheit für Mutter und Kind erhöht, sind im Wesentlichen drei Punkte:

1. Schaffung von fachlicher Kompetenz

2. Training von Notfallsituationen

3. Effiziente Fehleranalyse

\section{Schaffung von fach- licher Kompetenz}

Wesentlich für eine effiziente Risikostratifizierung ist das Vorhandensein von hausinternen Leitlinien (SOPs, Standard Operating Procedures).

Der Leitlinienordner muss in jedem Kreißsaal an einer gut sichtbaren Stelle vorhanden sein. Er soll die Behand- 
lungskonzepte der wichtigsten Risikosituationen beinhalten. Die Anleitungen sind so konkret zu halten, dass auch ein fachlich noch nicht so versierter Mitarbeiter das optimale Therapiekonzept einleiten kann. Neben der medikamentösen Therapie sind organisatorische und interdisziplinare Abläufe mit den entsprechenden Telefonnummern in den SOPs hinterlegt.

Ein Leitlinienordner für ein standardisiertes Vorgehen ist jedoch auch für die Betreuung im Geburtshaus, z.B. für eine Verlegung im Notfall, erforderlich.

\section{Training von Notfallsituationen}

Notfallsituationen, die sich für ein geburtshilfliches Simulationstraining eignen, sind:

- Fetale Bradykardien

- Peripartale Blutungen

- Schulterdystokien

- kindliche Erstversorgung

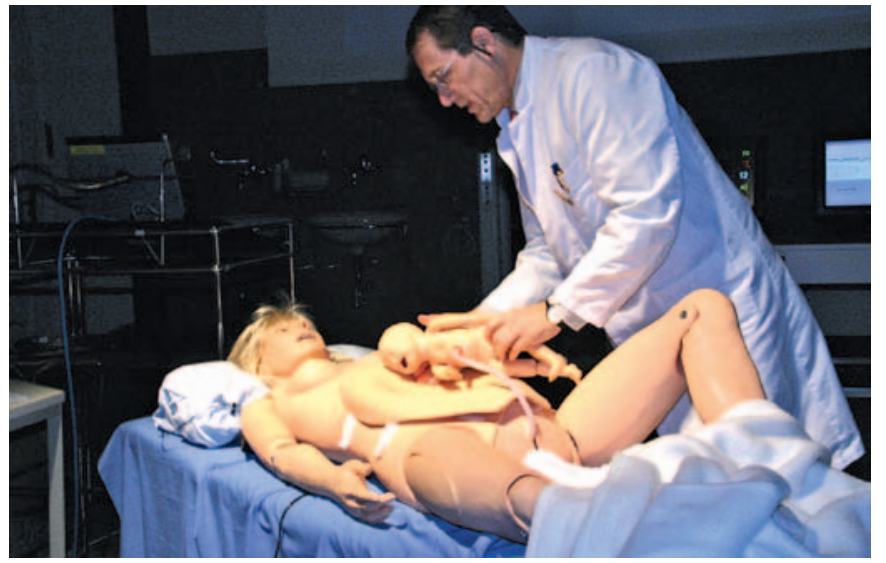

Abb. 1: Simulationspuppe nach Spontangeburt.

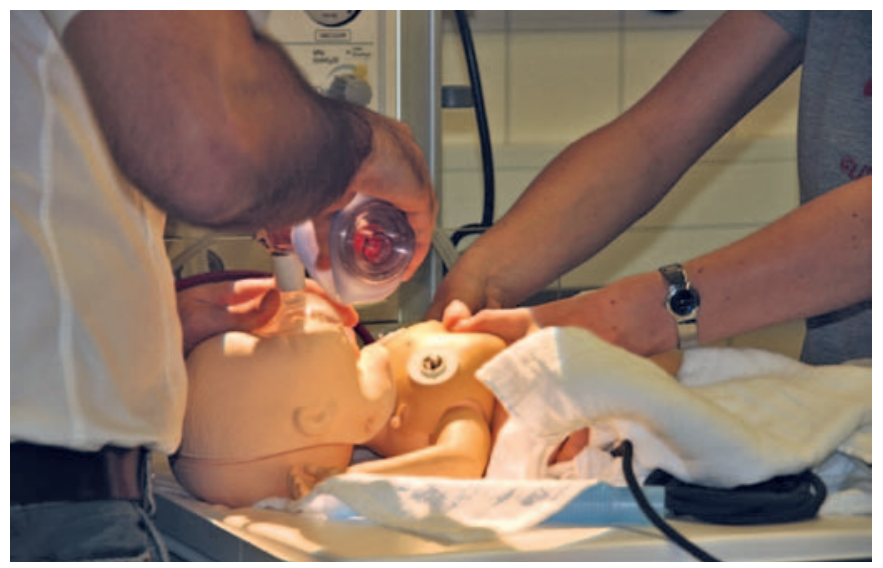

Abb. 2: Training der Erstversorgung anhand einer Neugeborenensimulationspuppe. 


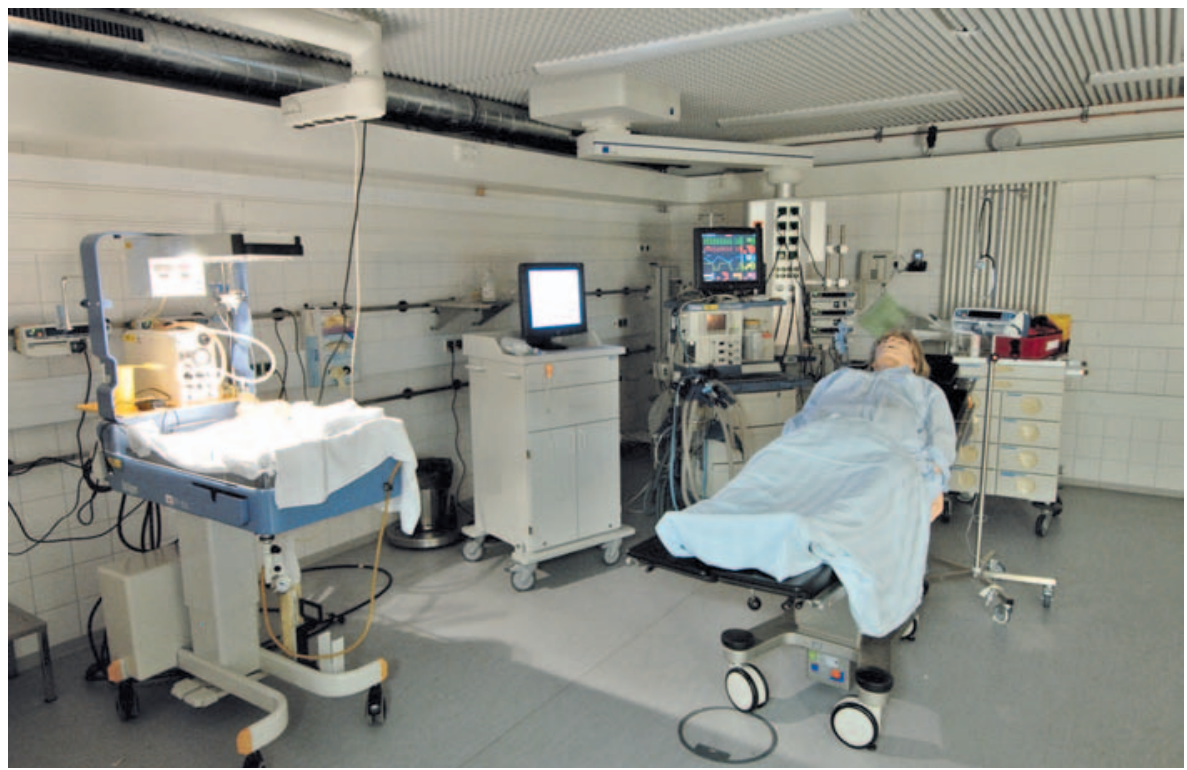

Abb. 3: Simulationskreißsaal des Instituts für Intensivmedizin und Medizinmanagement (INM) der Ludwig-Maximilian-Universität München.

- Präeklampsien und Eklampsie

- Fruchtwasserembolien

- Nabelschnurvorfälle

- Uterusrupturen

- Notsectiones

Aber auch Abläufe im Geburtshaus oder in der Hausgeburtshilfe können problemlos simuliert werden.

Das Training erfolgt optimalerweise in einem dafür eingerichteten Simulationskreißsaal. Dabei stehen dem Team sämtliche Therapiemöglichkeiten eines Kreißsaales zur Verfügung. Für eine realitätsnahe Darstellung des Geburtsverlaufes stehen lebensgroße Simulationsmodelle zur Verfügung, bei denen die üblichen Vitalparameter (Blutdruck, Puls, Atmung, Sauerstoffsättigung) beurteilt werden können. Medikamente können intravenös verabreicht werden. Bei einigen Puppen ist auch eine verbale Kommunikation möglich.

Die Simulation der Geburt erfolgt entweder über einen Motor oder die Puppe wird manuell von einer Hilfsperson durch das Becken geschoben. Die Steuerung der Simulationspuppe erfolgt kabellos aus einem Regieraum mit online-
Monitoring des CTG-Verlaufes. Zusätzlich steht ein Neugeborenenreanimationsplatz mit einer speziellen Babypuppe zur Verfügung, bei welcher die Apgarwerte vom Regieraum aus „eingestellt" werden können (Abb. 1 bis 3).

Geburtsmodelle: „575 Noelle“ (Firma Gaumard), „PROMPT, MamaNatalie, SimMom, SimBaby, NewbornHAl“ (Firma Laerdal, Stavanger).

Der Geburtsablauf ist dem Team nicht bekannt und dauert ca. 15 Minuten. Der gesamte Geburtsablauf wird von Instruktoren (meist Hebamme, Geburtshelfer, Anästhesist und Techniker) aus dem Regieraum gesteuert, der von den Teilnehmern nicht einsehbar ist. Das Geburtsszenario wird von Videokameras dokumentiert, ebenso erhält jeder Teilnehmer ein Mikrophon, sodass sämtliche Gespräche aufgezeichnet werden können.

Für effektives Training ist jedoch nicht unbedingt ein hochtechnisierter Simulationskreißsaal die Voraussetzung. Die Geburtssituation kann auch im hauseigenen Kreißsaal oder als Hausgeburt dargestellt werden. Dabei werden die Simulationsmodelle durch „Hybrid- simulationen“ (Darstellung der Gebärenden durch Schauspieler) ersetzt. Die Geburt kann dabei mit kostengünstigen Geburtsmodellen (MamaNatalie ${ }^{\circledR}$ Birth Simulator) simuliert werden.

\section{Effiziente Fehleranalyse}

Nach dem Ablauf des Trainingsszenariums werden in einer Nachbesprechung die Abläufe analysiert. Es wird offen über Fehlentscheidungen gesprochen, ohne die Teilnehmer öffentlich zu blamieren. Die wesentlichen Punkte wie Entscheidungsfindung, Kommunikation sowie Teamarbeit werden nochmals angesprochen.

Bei Fehlentscheidungen wird versucht, die tatsächliche Ursache für den fehlerhaften Ablauf zu eruieren. Dabei lernen die Teilnehmer, dass Fehler im Kreißsaal mit schwerwiegenden Komplikationen fast nie individuell verursacht werden, sondern dass in den meisten Fällen erst eine Verkettung von mehreren fehlerhaften Abläufen die Katastrophe auslöst.

Eine individuelle Fehlerzuordnung ist daher in den meisten Fällen nicht zielführend.

\section{Fazit}

In der Anästhesie und Notfallmedizin ist das Simulationstraining seit vielen Jahren bereits etabliert. In den letzten 10 Jahren wird das Training auch in der Kinderheilkunde zunehmend eingesetzt. Im deutschsprachigen Raum wird das Simulationstraining in der Geburtshilfe seit etwa 5 Jahren an Zentren angeboten.

Nach meiner knapp 10-jährigen Erfahrung mit Notfalltraining im Simulationskreißsaal kann ich folgendes Fazit ziehen: 
- Eine unzureichende Kommunikation und eine nicht klar geregelte Aufgabenverteilung zwischen Hebammen, Geburtshelfern, Neonatologen sowie Anästhesisten kann auch bei optimalen medizinischen Möglichkeiten in einem Zentrum in vielen Fällen die Ursache für eine suboptimale Behandlung der Patienten sein.

- Außer den tragischen, meist vermeidbaren, gesundheitlichen Konsequenzen für Mutter und Kind aus geburtshilflichen Fehlentscheidungen sprechen auch die extrem hohen finanziellen Schadenssummen für ein Training von Notfallsituationen.

- Neben der Reduzierung von fachlichen Fehlern führt die Simulation zu einer Verbesserung der Kommunikation und der Teamarbeit, da sie den Teilnehmern einen guten Einblick in die Denk- und Vorgehensweise der Nachbardisziplinen (Hebammenarbeit, Anästhesie, Intensivmedizin, Neonatologie) ermöglichen.

- Die Evaluierung der bisherigen Trainings hat gezeigt, dass die Teilnehmer mit dem Training hoch zufrieden waren und viele wertvolle Erfahrungen für den klinischen Alltag mitnehmen konnten.

- Kosten-Nutzen-Relation: Die Einrichtung eines optimalen Simulationszentrum ist finanziell nur von großen Abteilungen umsetzbar. Es sind jedoch auch kostengünstige lokale Trainingsmöglichkeiten sinnvoll. In Relation zu einem Schadensfall, der neben den medizinischen Kosten auch forensisch mehrere Millionen Euro Schadensersatz verursachen kann, sind die Kosten für ein Simulationstraining vernachlässigbar.

Das geburtshilfliche Simulationstraining wird deshalb in Zukunft mit großer Wahrscheinlichkeit einen hohen Stellenwert in der Ausbildung von Hebammen, Geburtshelfern, Anästhesisten und Kinderärzten einnehmen.

\section{Literatur}

1 Draycott $\mathrm{T}$ et al. Improving neonatal outcome through practical shoulder dystocia training. Obstet Gynaecol 2008; 112: 14-20

2 Jude DC, Gilbert GG, Magrane D. Simulation training in the obstetrics and gynecology clerkship. Am J Obstet Gynecol 2006; 195: 1489-1492

3 Kainer F et al. Simulation in der Geburtshilfe. In: Simulation in der Medizin, St. Pierre, Breuer (Hrsg.), Springer Verlag Berlin Heidelberg 2013

\section{Anschrift des Autors:}

Prof. Dr. Franz Kainer

Chefarzt der Abteilung Geburtshilfe und Neonatologie Klinikum Wallerwiese St. Johannis Mühlgasse 19 90419 Nürnberg E-Mail: Franz.Kainer@ diakonieneuendettelsau.de 